\title{
Desarrollo integral de procesos de adaptación al cambio en pequeñas y medianas empresas
}

\author{
Carlos Pacheco-Ruíz ${ }^{1}$, Claudia Rojas-Martínez ${ }^{1}$, William Niebles-Nuñez ${ }^{1}$ y Hugo G. Hernández-Palma ${ }^{2}$ \\ (1) Universidad de Sucre, Programa de Administración de Empresas, Sincelejo-Colombia. \\ (correo-e: carlos.pacheco@unisucre.edu.co; claudia.rojas@unisucre.edu.co; william.niebles@unisucre.edu.co) \\ (2) Universidad del Atlántico, Programa Administración de Empresas, Barranquilla-Colombia. \\ (correo-e: hugohernandezpalma@gmail.com)
}

Recibido Feb. 18, 2020; Aceptado Abr.21, 2020; Versión final May. 22, 2020, Publicado Oct. 2020

\begin{abstract}
Resumen
El presente estudio estuvo orientado al análisis del desarrollo integral de procesos de adaptación al cambio en pequeñas y medianas empresas (pymes) de servicios tecnológico. Esta es una investigación descriptiva no experimental de campo. La población estuvo integrada por 44 directivos, a quienes se les aplicó una encuesta con escala tipo Likert, constituida con 19 afirmaciones referidas a cultura y cambio organizacional. Este instrumento fue sometido a la validez de contendido y se aplicó el coeficiente de confiablidad de Alfa Cronbach, arrojando un coeficiente de 0,85. El análisis de los resultados se presentó a través de tablas de frecuencias. Se concluye que los procesos ejecutados para la adaptación al cambio se sustentan en una cultura organizacional fuerte, lo cual induce a que exista resistencia. Esto genera la necesidad de promover programas de desarrollo organizacional que disminuya la resistencia al cambio, así como, romper con viejas costumbres dando paso a nuevas tecnologías.
\end{abstract}

Palabras clave: cambio organizacional; cultura; resistencia al cambio; procesos; adaptación

\section{Integral development of adjustment processes towards change in small and medium size enterprises}

\begin{abstract}
The present study aimed to analyze the integral development of processes adapting to change in small and medium size enterprises (SMEs) of technological services. This study is a descriptive non-experimental field investigation. The population (44 managers) was surveyed with a Likert scale questionaire, consisting of 19 statements relating to culture and organizational change. This instrument was subjected to the validity of contention. The coefficient of trust of Alfa Cronbach was applied yielding a coefficient of 0.85 . The analysis of the results was presented through frequency tables. It is concluded that the processes executed for adaptation to change are based on a strong organizational culture, which induces resistance. This generates the need to promote organizational development programs, which diminish resistance to change and breaks with old customs, giving way to new technologies.
\end{abstract}

Keywords: organizational change; culture; resistance to change; processes; adaptation 


\section{INTRODUCCIÓN}

En la actualidad los cambios tecnológicos, sociales y económicos han creado la necesidad en las organizaciones de generar planes de desarrollo que le permitan competir con el mercado global, por lo cual deben contar con un personal capacitado para adaptarse al entorno, y cumplir con éxito los objetivos establecidos por la alta gerencia. En este sentido, es primordial para las empresas modificar sus rígidos esquemas de trabajo por otras flexibles que promuevan la participación del personal, en las acciones empresariales. Estas se encuentran inmersas en un proceso de globalización, el cual genera la necesidad de identificar fortalezas y debilidades, por ende, tiende a desarrollar estrategias para lograr competir con efectividad y calidad en los mercados nacionales e internacionales. Bajo este contexto, Valenzuela et al (2018), al igual que Simancas et al (2018), sostienen que el recurso humano representa uno de los factores más importante en la gestión empresarial, por tal razón los cambios estratégicos deben estar apoyados por los valores, actitudes y conducta de su gente, en virtud de promover el trabajo en equipo de forma efectiva, que permitan alcanzar los objetivos organizacionales. Dichos cambios se generan desde el análisis de su estructura, incluyendo el estudio de su cultura organizacional, con el fin de adaptarse a los nuevos paradigmas o procesos en el mercado.

En este ámbito empresas de servicio, por su misma dinámica diaria, tratan los cambios como algo que ocurre de manera accidental que involucra las actividades de negocio que son proactivas y con propósito. Por ende, para Moreno et al (2017), estratégicamente el cambio, según Hernández et al (2018), debe gestarse como una actividad intencional orientada hacia la meta, dado que el ambiente que envuelve a esta organización es demasiado dinámico, lo cual exige una elevada capacidad de adaptación como condición básica de supervivencia. En este sentido, los individuos asumen un determinado comportamiento ante los cambios producidos dentro de la Organización; estos para Hernández, H. et al (2017), tienen su origen en el medio ambiente donde se desarrolla el empleado, así como también están relacionados con las creencias y valores que son manejados en la organización, todo esto se puede definir en dos palabras "cultura organizacional", la cual según Marulanda et al (2018), refieren que el conjunto de las nociones importantes (tales como, normas, valores, actitudes y creencias), que comparten los miembros de la organización. Por otro lado, Robbins y Judge (2017), la plantea, como un sistema de significado compartido entre sus miembros, y que distingue a una organización de las otras, dentro de la cual se resumen siete características principales que, en conjunto, captan la esencia de la cultura de una organización: Innovación y asunción de riesgo, atención al detalle, orientación a los resultados, orientación hacia las personas, orientación al equipo, energía, y estabilidad, Cradazco et al (2019). Asimismo, la cultura organizacional para Osorio et al (2018), involucra el ambiente laboral en el cual los empleados de una organización desarrollan su trabajo. Es algo no visible, ni se puede tocar, por lo tanto, afecta directamente todo lo ocurrido en la empresa, asimismo representa un factor determinante para la constante dinámica de cambio, la cual comprende niveles organizacionales, de esta manera se puedan efectuar los procesos de cambio requeridos por la organización, estimados por Parra et al (2019).

Ciertamente, la cultura organizacional es concebida como la manera en la cual cada organización ejecuta sus actividades, precisando la interrelación de varios elementos involucrados en su creación; estos son: las costumbres, tradiciones, y la forma en general de cómo la empresa cumple con sus políticas. Según Viloria, et al (2019), existen diversas formas de transmitir la cultura, algunas de estas se relacionan con las virtudes, símbolos materiales, el lenguaje además el desempeño del trabajo en equipo; lo cual influye en los cambios organizacionales. Por otro lado, la cultura es una desventaja, Bravo et al (2017) sostienen que cuando los valores compartidos no van de acuerdo con los que harán progresar la eficacia de la organización, por lo cual las conductas o actitudes de los trabajadores son establecidas por los paradigmas existentes, estos no permiten aplicar un esfuerzo positivo para implantar el cambio.

Al respecto, French et al (2005), sostienen, "el proceso de cambio organizacional comienza con la aparición de fuerzas que vienen de afuera o de algunas partes de la organización. Las mismas pueden ser exógenas o endógenas a la organización. Las fuerzas exógenas provienen del ambiente, como las nuevas tecnologías, los cambios en los valores de la sociedad y las nuevas oportunidades o limitaciones del ambiente (económico, político, legal y social). Dentro de este marco, Pierce et al (2013) cita a Lewin con su modelo de campo de fuerzas y manifiesta que las fuerzas externas crean la necesidad de cambio organizacional interno, dichos intentos de cambio pueden planearse conscientemente para ajustarse a las nuevas condiciones externas, realizándose con una mínima perturbación del equilibrio estructural y del comportamiento existente dentro de la organización. Así es como las fuerzas endógenas crean la necesidad del cambio estructural, del mismo modo las fuerzas del comportamiento provienen de la tensión organizacional: tensión de las actividades, interacciones, sentimientos o resultados del desempeño en el trabajo. En este sentido, para De la Hoz J. et al. (2020), el cambio debe iniciarse desde la alta gerencia e involucrar las áreas de la organización para de esta manera vencer la incertidumbre o el miedo a lo desconocido. Un proceso de cambio ocurre de forma eficiente si los miembros están comprometidos con él. 
En tanto, García et al (2018), enunciaron que las personas se comprometan, ellas no pueden ser atropelladas por el proceso, como si fueran algo alejada del mismo, en realidad, el cambio ocurre a través de las personas, porque al hablar de cambio se enfoca en un conjunto de transferencias, reorganización, ampliación o reducción de actividades, nuevos departamentos, productos, servicios, mercados, tecnologías, estos cambios son implantados con la finalidad de mejorar los procesos existentes considerando las personas como parte del proceso de cambio es necesario conocer sus valores, creencias, sus comportamientos. Por ende, en las organizaciones modernas se están originando cambios, como parte de los sistemas que se ven afectados por los procesos orientados a la toma de decisiones radicales, por lo cual los gerentes y trabajadores del nuevo milenio deben poseer las destrezas y habilidades necesarias para enfrentar dichos cambios, minimizado de manera eficiente los efectos producidos en las empresas. Es así como la tolerancia al cambio representa un papel importante para el desarrollo y crecimiento de la organización, permitiendo su permanencia en el mercado, generando mayores niveles de rentabilidad y productividad.

Bajo este contexto, se presentan las empresas de servicios tecnológicos de Región Caribe, las mismas iniciaron sus operaciones bajo una filosofía de gestión familiar ofreciendo productos a un grupo determinado, sin un proceso planificado ni estructurado con visión a futuro. No obstante de acuerdo al creciente desarrollo que ha tenido la Región Caribe, estas organizaciones se han hecho consciente de las transformaciones que ofrece el nuevo milenio, donde se ha venido desarrollando un trabajo de integración económica y crecimiento de la infraestructura, logrando un mayor desarrollo social y económico, buscando cerrar la brecha regional que afecta a esta área de Colombia, a tal punto que para el último cuatrienio, hubo un aumento de las inversiones en el Plan Nacional de Desarrollo, que resultaron en COP $\$ 182$ billones. Por lo cual ha surgido la necesidad de adaptarse al mercado competitivo, para ello requieren implantar cambios organizacionales (administrativos, estructurales y sistemáticos), estos cambios traen como consecuencia la necesidad de realizar estudios sobre los valores, actitudes y motivación de los empleados que afectan los procesos en las diferentes unidades organizativas, producidas por los procesos de cambio. La principal problemática radica de la cultura de los gerentes, la cual no permite adoptar nuevas técnicas de trabajo, ni la participación de los empleados en la toma de decisiones, como una manera viable de generar aportes, destacándose la cultura organizacional como uno de los componentes que afectan los procesos; esta es transmitida a través de sus fundadores a los gerentes o trabajadores, por ende la cultura existente en la empresa está enmarcada con paradigmas arraigados a las actitudes, valores y prioridades en la organización, afectando el desarrollo evolutivo de los procesos organizacionales, aunado al del empleado.

Por otra parte, se ha determinado que la cultura existente establece ciertas limitaciones, en cuanto al adiestramiento del personal, la vinculación a la filosofía de gestión, no se están aplicando técnicas de atención asertiva al cliente, no promueven el trabajo en equipo, por otra parte, las fuerzas externas a la empresa no permiten cubrir las necesidades del cliente, dado que hay limitaciones en algunas zonas para acceder a estos lugares de servicio al cliente. De este punto parte la inquietud de realizar un proyecto en Empresa de servicios específicamente en el mercado de empresas de servicios tecnológicos, surge entonces la necesidad de determinar la relación entre cambio organizacional y estilo cultural predominante en empresa de servicios tecnológicos de la Región Caribe.

\section{OTROS ANTECEDENTES}

El cambio no es un factor ajeno a la realidad de las organizaciones, es un elemento inherente a sus procesos, el cual requiere de los altos directivos y gerentes una respuesta para satisfacer las necesidades producidas por tales alteraciones, en termino de eficiencia, eficacia y un compromiso con la organización de promover las ventajas que los cambios plantean. El mundo actual se caracteriza por un cambio constante en el ambiente donde se desarrollan las organizaciones y es demasiado dinámico, lo cual exige una elevada capacidad de adaptación como condición básica de supervivencia.

En la sociedad actual, el cambio es constante y todos se ven afectados por él. Como agentes de cambio, los individuos que participan en el desarrollo de los recursos humanos deben entender la secuencia del cambio, las dificultades que trae y las formas para reducir la resistencia al mismo. Por otra parte, Pierce et al (2013), refieren que el cambio es cualquier alteración ocurrida en el entorno de trabajo. Sin duda, el cambio es una estrategia de desarrollo que le permitirá a las organizaciones hacerse competitivos ante las expectativas de una globalización mundial que afecta directamente las estructuras de estas. La secuencia del cambio no termina cuando el mismo se ha implantado. Se debe desarrollar una posición nueva y flexible, capaz de enfrentar las exigencias actuales y adaptarse a los cambios adicionales. Al respecto Mondy et al (2016), plantean que el cambio en virtud de su impacto sobre la organización y sus empleados debe emprenderse sólo cuando es verdaderamente necesario. Desde luego, determinadas circunstancias en los ambientes interno y externo pueden hacer que el cambio sea deseable o necesario. El ímpetu básico para promoverlo proviene de la creencia de que la organización y sus recursos humanos pueden ser más productivo y tener mayor éxito después del cambio. 
La reducción de la resistencia al cambio es importante para el éxito. En ocasiones, esto puede ser muy difícil porque puede exigir también cambios en las actitudes. Sin embargo, si se reduce o se elimina la resistencia, se puede implantar el cambio con mayor efectividad. Este puede surgir de una orden o una sugerencia, o se puede emprender de manera voluntaria. El cambio se desarrollará más satisfactoriamente si la persona afectada lo desea y lo cree necesario. El poder cambiar una actitud exige confianza y respeto entre las personas que tratan de implantar el cambio y los individuos afectados por el mismo Ímpetu. Como ya se mencionó, una fuente principal de efectividad, lo que para Jiménez et al (2018), es la forma en que el programa afecta a los empleados de menor nivel de la compañía. El desarrollo de recursos humanos es un proceso continuo, porque tanto el ambiente interno como el externo de la organización son dinámicos y siempre inciden en el statu quo. Las organizaciones encuentran en su camino muchas fuerzas que las inducen al cambio. Estas fuerzas provienen de fuentes externas ajenas a la organización y de fuentes internas a la misma. En efecto, Chiavenato (2015), dice que el proceso de cambio organizacional comienza con la aparición de fuerzas que vienen de fuera o de algunas partes de la organización. Esas fuerzas, dice, pueden ser exógenas o endógenas a la organización.

Las fuerzas exógenas provienen del ambiente, como las nuevas tecnologías, según Tarhini et al (2015), los cambios en los valores de la sociedad y las nuevas oportunidades o limitaciones del ambiente (económico, político, legal y social). Esas fuerzas externas crean la necesidad de un cambio organizacional interno. Los intentos de cambio interno pueden planearse conscientemente para que el ajuste a las nuevas condiciones externas se realice con una mínima perturbación del equilibrio estructural y del comportamiento existente dentro de la organización. Las fuerzas endógenas que crean la necesidad del cambio estructural y del comportamiento provienen de la tensión organizacional: tensión de las actividades, interacciones, sentimientos o resultados del desempeño en el trabajo. Por su parte, Kreitner y Kinicki (2006), refieren que las fuerzas externas para el cambio son las que tienen su origen fuera de la organización. Dado que estas fuerzas tienen efectos globales, pueden hacer que una organización se pregunte sobre la naturaleza del negocio al que se dedica y sobre el proceso utilizado para la producción de bienes y servicios. Se han identificado cuatro fuerzas externas que son básicas para el cambio: características demográficas, progresos tecnológicos, cambio en los mercados y presiones sociales y políticas.

\section{METODOLOGÍA}

El tipo de investigación se relaciona con la naturaleza de la problemática en estudio, con los objetivos que se pretenden alcanzar, en este sentido este estudio se cataloga como descriptivo, ya que está orientado a recolectar información relacionada con el estado real del cambio y cultura organizacional en empresas del sector tecnológico tal como se percibe en su estado natural. Para Hernández, et al (2017), los estudios descriptivos buscan especificar las propiedades importantes de personas grupos, comunidades o cualquier otro fenómeno que sea sometido a análisis. Miden o evalúan diversos aspectos, dimensiones o componentes del fenómeno a investigar. Además, el mismo se basa en una investigación de campo, porque el estudio se ejecutó en las instalaciones de tres empresas del sector tecnológico lo que facilitó recolectar datos de una forma directa de la realidad, específicamente en el área seleccionada para ello. En el mismo orden de ideas, se presenta el diseño, el cual se clasifica como no experimental debido a que en ningún momento se pretendió manipular las variables en estudio (cultura y cambio organizacional), por otra parte, es transversal porque fue medido en un momento único y determinado.

Atendiendo a otras consideraciones, se distribuye la población, para la mejor interpretación de los resultados, la población estuvo conformada por directivos y trabajadores generales, así como empleados administrativos de las empresas seleccionadas. En total se tuvo una población de 44 personas, 34 fueron empleados administrativos y 10 directivos y gerentes de los distintos departamentos administrativos de las empresas seleccionadas, a los cuales se les realizó encuestas cara a cara. Según las características que presenta la población: pertenecientes al nivel supervisor, con más de 3 años en la empresa y mayores a los 40 años, se consideró la utilización del censo poblacional, que consiste en el análisis de la totalidad de los sujetos que constituyen la población, a fin de determinar su comportamiento ante un evento. Asimismo, en el presente estudio se recurrió a la técnica de la encuesta, la cual se emplea mediante herramientas, instrumentos o medios, que utiliza el investigador para medir el comportamiento o atributos de las variables. En el estudio se utilizó una encuesta estructurada con escala tipo Likert, cuyo contenido estuvo íntimamente relacionado con las interrogantes planteadas en la investigación, las cuales son: 1) ¿Cuáles son los elementos los elementos de la cultura organizacional presentes en las empresas?; 2) ¿Cuál es el estilo de cultura organizacional predominante en las empresas?; 3) ¿Como serán las técnicas para superar la resistencia al cambio en las empresas?

Consecutivamente, todo instrumento se le debe aplicar la validez de contenido, Para validar el instrumento se seleccionó a un grupo de diez (10) expertos en el área de gerencia empresarial, administración, así como en metodología, con la finalidad de solicitar su opinión y aprobación con relación a: grado de correspondencia 
de las variables con los objetivos de la investigación, así como con las dimensiones e indicadores estudiados e ítems derivados. En este sentido, los expertos realizaron algunas observaciones en la redacción de ciertos ítems que presentaron dificultad o ambigüedad, estos fueron corregidos para la redacción final del instrumento. Posteriormente se requería calcular la confiabilidad de los cuestionarios diseñados, se seleccionó el coeficiente de Alfa Cronbach, el cual se aplica en una sola administración del instrumento de medición, representado por valores entre 0 y 1 . Esta expresión se aplicó a la prueba piloto conformada por treinta y dos sujetos (32) que poseían características similares a las de la muestra definitiva, los valores obtenidos establecen unos intervalos que oscilan entre 0 y 1 entendiéndose el coeficiente 0 como nula confiabilidad y 1 como confiabilidad total. De acuerdo con los resultados obtenidos, para ambos instrumentos referidos a Cultura organizacional y cambio fue de 0.85 considerándose dentro del rango de altamente confiables.

\section{RESULTADOS Y DISCUSIÓN}

Los resultados ilustrados en la Tabla 1 evidencian que el $48 \%$ de la muestra manifestó que casi siempre en las actividades de la organización se toma en cuenta la participación de los trabajadores, mientras que el $20 \%$ contestó casi nunca, en tanto el $16 \%$ opto por la opción siempre, y el mismo porcentaje respondió nunca, como resultado se obtuvo que la mayoría de los trabajadores consideran que son tomados en cuenta para la toma de decisiones.

Tabla 1. Participación del personal

\begin{tabular}{|l|l|l|}
\hline Opciones & F/A & $\%$ \\
\hline Siempre & 7 & 16 \\
\hline Casi siempre & 21 & 48 \\
\hline Nunca & 7 & 16 \\
\hline Casi nunca & 9 & 20 \\
\hline Total & 44 & 100 \\
\hline
\end{tabular}

Luego se tiene que al personal se les pregunto si la labor desempeñada por los trabajadores se rige bajo las normas existentes en la organización, y las respuestas obtenidas fueron: el $48 \%$ de los encuestados respondió la opción de siempre, al tiempo que el $45 \%$ optó por casi siempre, mientras que el $5 \%$ expresó que nunca, y el $2 \%$ casi nunca. Como se puede apreciar la gran mayoría de los encuestados considera que la labor que desempeñan se rige por las normas de la organización. Estos datos son expuestos a continuación en la Tabla 2.

Tabla 2. Normas existentes en la organización

\begin{tabular}{|l|l|l|}
\hline Opciones & $F / A$ & $\%$ \\
\hline Siempre & 21 & 48 \\
\hline Casi siempre & 20 & 45 \\
\hline Nunca & 02 & 05 \\
\hline Casi nunca & 01 & 02 \\
\hline Total & 44 & 100 \\
\hline
\end{tabular}

En cuanto a la aceptación de las políticas implementadas por la empresa, los resultados (expresados en la Tabla 3) obtenidos fueron: el $62 \%$ opinó siempre, el $25 \%$ la opción casi siempre, $11 \%$ expreso que casi nunca se aceptan estas políticas y el $2 \%$ respondió que nunca sucede este hecho, al respecto de estos resultados se deduce que la mayoría de los encuestados aceptan las políticas implementadas por la gerencia dentro de la organización.

Tabla 3. Aceptación de las políticas de la Organización

\begin{tabular}{|l|l|l|}
\hline Opciones & F/A & $\%$ \\
\hline Siempre & 27 & 62 \\
\hline Casi Siempre & 11 & 25 \\
\hline Nunca & 01 & 02 \\
\hline Casi Nunca & 05 & 11 \\
\hline Total & 44 & 100 \\
\hline
\end{tabular}

Con respecto a la interrogante sobre si los trabajadores comparten los valores organizacionales de la empresa, en la Tabla 4, se reflejan los resultados de la siguiente manera, un $36 \%$ opinó casi siempre, por otro lado, el $36 \%$ consideró casi nunca, mientras que el 14\% contestó nunca y un $14 \%$ respondió siempre, en este sentido, se deduce que la población encuestada no se siente totalmente identificada con los valores de la organización. 
Tabla 4. Comparten los trabajadores los valores organizacionales

\begin{tabular}{|l|l|l|}
\hline Opciones & F/A & $\%$ \\
\hline Siempre & 06 & 14 \\
\hline Casi siempre & 16 & 36 \\
\hline Nunca & 06 & 14 \\
\hline Casi nunca & 16 & 36 \\
\hline Total & 44 & 100 \\
\hline
\end{tabular}

Al personal encuestado se le cuestionó sobre si la empresa le ofrece elementos motivacionales que le permitan identificarse con ella, para ello sus respuestas fueron las siguientes: el 34\% respondió casi siempre, mientras que el $27 \%$ manifestó que nunca reciben este tipo de elementos, en tanto el $23 \%$ contestó que esto siempre sucede y el $16 \%$ expresó casi nunca, lo cual evidencia que la mayoría de los encuestados consideran que existen elementos motivacionales, que le permiten identificarse con la empresa. La Tabla 5 muestra los resultados a continuación.

Tabla 5. La empresa ofrece elementos motivacionales

\begin{tabular}{|l|l|l|}
\hline Opciones & F/A & $\%$ \\
\hline Siempre & 10 & 23 \\
\hline Casi siempre & 15 & 34 \\
\hline Nunca & 12 & 27 \\
\hline Casi nunca & 07 & 16 \\
\hline Total & 44 & 100 \\
\hline
\end{tabular}

Los resultados expuestos en la Tabla 6, responden a si las costumbres de la empresa están por encima de los trabajadores, donde el $59 \%$ respondió casi siempre, en tanto el $25 \%$ contestó siempre, mientras que en menor proporción de los encuestados respondió con las opciones negativas sobre el cuestionamiento con el $11 \%$ opinó nunca y el $5 \%$ manifestó que casi nunca. Estos resultados evidencias que la mayoría de la población encuestada considera que las costumbres de la empresa están por encima de las costumbres de los trabajadores.

Tabla 6. Están las costumbres de la empresa por encima de los trabajadores

\begin{tabular}{|l|l|l|}
\hline Opciones & $F / A$ & $\%$ \\
\hline Siempre & 11 & 25 \\
\hline Casi siempre & 26 & 59 \\
\hline Nunca & 5 & 11 \\
\hline Casi nunca & 2 & 5 \\
\hline Total & 44 & 100 \\
\hline
\end{tabular}

En la Tabla 7, se aprecia la información con respecto a si los trabajadores comparten las costumbres establecidas en la empresa, y los resultados obtenidos para esto fueron los siguientes: $59 \%$ de la muestra manifestó estar de acuerdo, mientras que el 16\%, opinó estar en desacuerdo en tanto el 14\% expresó estar totalmente de acuerdo y el $11 \%$ contestó estar en desacuerdo, en este sentido se concluye que la mayoría de los trabajadores representada por casi el $75 \%$ de los encuestados comparten las costumbres establecidas en la empresa.

Tabla 7. Comparten los trabajadores las costumbres establecidas en la empresa

\begin{tabular}{|l|l|l|}
\hline Opciones & $F / A$ & $\%$ \\
\hline $\begin{array}{l}\text { Totalmente de } \\
\text { acuerdo }\end{array}$ & 6 & 14 \\
\hline De acuerdo & 26 & 59 \\
\hline En desacuerdo & 7 & 16 \\
\hline $\begin{array}{l}\text { Totalmente en } \\
\text { desacuerdo }\end{array}$ & 5 & 11 \\
\hline Total & 44 & 100 \\
\hline
\end{tabular}

En lo que respecta al compromiso adquirido por los trabajadores para el cumplimiento de los objetivos organizacionales, se obtuvo el siguiente resultado: el $43 \%$ del personal manifestó que siempre existe un compromiso para el cumplimiento de los objetivos, en tanto el $41 \%$ respondió casi siempre, por otro lado el $14 \%$ expresó nunca y finalmente el $2 \%$ contestó casi nunca, lo cual evidencia que ciertamente existe un compromiso adquirido por los trabajadores para el cumplimiento de los objetivos organizacionales. Estos datos son mejor apreciados en la Tabla 8. 
Tabla 8. Existe un compromiso de los trabajadores para el cumplimiento de los objetivos.

\begin{tabular}{|l|l|l|}
\hline Opciones & $F / A$ & $\%$ \\
\hline Siempre & 19 & 43 \\
\hline Casi siempre & 18 & 41 \\
\hline Nunca & 6 & 14 \\
\hline Casi nunca & 1 & 2 \\
\hline Total & 44 & 100 \\
\hline
\end{tabular}

Los datos expresados en la Tabla 9 muestran los resultados acerca de la identificación de los trabajadores con la razón de ser de la organización, donde el 59\% del personal respondió casi siempre, mientras que el $18 \%$ contestó siempre, por otro lado, el 16\% opinó casi nunca y el 7\% expresó inclinación por la opción nunca. En líneas generales la mayor parte de la población se identifica con la razón de ser de la organización.

Tabla 9. Identificación de los trabajadores con la razón de ser de la empresa

\begin{tabular}{|l|l|l|}
\hline Opciones & $F / A$ & $\%$ \\
\hline Siempre & 8 & 18 \\
\hline Casi siempre & 26 & 59 \\
\hline Nunca & 3 & 7 \\
\hline Casi nunca & 7 & 16 \\
\hline Total & 44 & 100 \\
\hline
\end{tabular}

Luego en la Tabla 10 se presenta que al personal se les preguntó si es posible que los patrones de conducta en la organización sean cambiados con facilidad y las respuestas fueron las siguientes: el 43\% opinó estar en desacuerdo, mientras que el 30\% respondieron de acuerdo, en tanto el 16\% expresó estar totalmente de acuerdo y finalmente el $12 \%$ manifestó estar totalmente en desacuerdo. Lo cual evidencia que los patrones de conducta no pueden ser cambiados con facilidad.

Tabla 10. Cambio en los patrones de conducta de la organización

\begin{tabular}{|l|l|l|}
\hline Opciones & $F / A$ & $\%$ \\
\hline Totalmente de acuerdo & 7 & 16 \\
\hline De acuerdo & 13 & 30 \\
\hline En desacuerdo & 19 & 43 \\
\hline $\begin{array}{l}\text { Totalmente en } \\
\text { desacuerdo }\end{array}$ & 5 & 11 \\
\hline Total & 44 & 100 \\
\hline
\end{tabular}

Sobre la adaptación de los trabajadores al cambio en los procesos, en la Tabla 11 se encuentran los resultados obtenidos: el 64\% manifestó que casi siempre se adaptan al cambio en la empresa, mientras que el $18 \%$ opinó siempre, el $11 \%$ contestó nunca y el $7 \%$ respondió casi nunca. Lo cual evidencia que los trabajadores se adaptan con facilidad al cambio en los procesos postulados por la empresa.

Tabla 11. Adaptación al cambio en los procesos

\begin{tabular}{|l|l|l|}
\hline Opciones & $F / A$ & $\%$ \\
\hline Siempre & 8 & 18 \\
\hline Casi siempre & 28 & 64 \\
\hline Nunca & 5 & 11 \\
\hline Casi nunca & 3 & 7 \\
\hline Total & 44 & 100 \\
\hline
\end{tabular}

También a los encuestados se les preguntó si se dictaban continuamente cursos de aprendizaje dentro de la empresa y las respuestas fueron: el $70 \%$ de la población manifestó que nunca les dictan cursos de aprendizaje, mientras que el $23 \%$ respondió casi nunca, por otro lado, el 5\% contestó casi siempre y finalmente el $2 \%$ respondió la opción de, siempre. Sin duda, no se dictan continuamente cursos de aprendizaje en las distintas empresas. Todo esto es apreciable en la Tabla 12.

Tabla 12. Se dictan cursos de aprendizaje en la empresa

\begin{tabular}{|l|l|l|}
\hline Opciones & $F / A$ & $\%$ \\
\hline Siempre & 1 & 2 \\
\hline Casi siempre & 2 & 5 \\
\hline Nunca & 31 & 70 \\
\hline Casi nunca & 10 & 23 \\
\hline Total & 44 & 100 \\
\hline
\end{tabular}


Con relación a si los trabajadores llevan a la práctica nuevas técnicas de trabajo, las respuestas fueron las siguientes: $51 \%$ del personal manifestó que solo a veces se llevan a la práctica las nuevas técnicas de trabajo, por otro lado, el $20 \%$ respondió casi nunca, mientras que el $18 \%$ opinó que siempre y el $11 \%$ contestó nunca. En virtud de los resultados obtenidos se concluye que la mayoría de los trabajadores llevan a la práctica las nuevas técnicas de trabajo. A continuación, la Tabla 13 presenta toda la información obtenida.

Tabla 13. Llevan a la práctica los trabajadores, nuevas técnicas de trabajo

\begin{tabular}{|l|l|l|}
\hline Opciones & $F / A$ & $\%$ \\
\hline Siempre & 8 & 18 \\
\hline Casi siempre & 22 & 51 \\
\hline Nunca & 5 & 11 \\
\hline Casi nunca & 9 & 20 \\
\hline Total & 44 & 100 \\
\hline
\end{tabular}

En los datos de la Tabla 14 se le preguntó al personal si se les permite realizar sugerencias para mejorar los procesos de trabajo, y los resultados de la encuesta fueron los siguientes: el 63\% respondió casi siempre, el $16 \%$ contestó casi nunca, en tanto el 14\% expresó siempre y el $7 \%$ opinó nunca. Estos resultados llevan a concluir que ciertamente se les permite a los trabajadores realizar sugerencias para mejorar los procesos de trabajo.

Tabla 14. Sugerencias para mejorar los procesos de trabajo

\begin{tabular}{|l|l|l|}
\hline Opciones & F/A & $\%$ \\
\hline Siempre & 6 & 14 \\
\hline Casi siempre & 28 & 63 \\
\hline Nunca & 3 & 7 \\
\hline Casi nunca & 7 & 16 \\
\hline Total & 4 & 100 \\
\hline
\end{tabular}

Al personal se les preguntó si los hábitos de trabajo generan resistencia al cambio y las respuestas fueron el $47 \%$ de la población encuestada expresó estar de acuerdo, el 30\% opinó estar totalmente de acuerdo y $23 \%$ manifestó estar desacuerdo. Estos resultados (presentados en la Tabla 15) evidencian que los hábitos de trabajo generan resistencia al cambio.

Tabla 15. Generan los hábitos de trabajo resistencia al cambio

\begin{tabular}{|l|l|l|}
\hline Opciones & F/A & $\%$ \\
\hline $\begin{array}{l}\text { Totalmente de } \\
\text { acuerdo }\end{array}$ & 13 & 30 \\
\hline De acuerdo & 21 & 47 \\
\hline En desacuerdo & 10 & 23 \\
\hline $\begin{array}{l}\text { Totalmente en } \\
\text { desacuerdo }\end{array}$ & 0 & 0 \\
\hline Total & 44 & 100 \\
\hline
\end{tabular}

En cuanto a si las costumbres obstaculizan el proceso de cambio, los resultados obtenidos fueron: $57 \%$ expresó estar de acuerdo, el 25\% opinó estar totalmente de acuerdo y el 18\% manifestó estar en desacuerdo. Estos resultados (expresados a continuación en la Tabla 16) indican que según la mayoría de la población encuestada las costumbres obstaculizan el proceso de cambio.

Tabla 16. Obstaculizan las costumbres el proceso de cambio

\begin{tabular}{|l|l|l|}
\hline Opciones & $F / A$ & $\%$ \\
\hline $\begin{array}{l}\text { Totalmente de } \\
\text { acuerdo }\end{array}$ & 11 & 25 \\
\hline De acuerdo & 25 & 57 \\
\hline En desacuerdo & 8 & 18 \\
\hline $\begin{array}{l}\text { Totalmente en } \\
\text { desacuerdo }\end{array}$ & 0 & 0 \\
\hline Total & 44 & 100 \\
\hline
\end{tabular}

Con relación a si el cambio en la empresa afectaría los puestos de trabajo, las respuestas expuestas en la Tabla 17 fueron que el $47 \%$ expresó estar en desacuerdo, mientras que el $25 \%$ manifestó estar de acuerdo, el $23 \%$ contestó estar totalmente de acuerdo y el $5 \%$ respondió totalmente en desacuerdo. En este sentido las evidencias demuestran que realmente un cambio en la empresa puede afectar los puestos de trabajo. 
Tabla 17. El cambio en la empresa afectaría los puestos de trabajo

\begin{tabular}{|l|l|l|}
\hline Opciones & $F / A$ & $\%$ \\
\hline $\begin{array}{l}\text { Totalmente de } \\
\text { acuerdo }\end{array}$ & 10 & 23 \\
\hline De acuerdo & 11 & 25 \\
\hline En desacuerdo & 21 & 47 \\
\hline $\begin{array}{l}\text { Totalmente en } \\
\text { desacuerdo }\end{array}$ & 2 & 5 \\
\hline Total & 44 & 100 \\
\hline
\end{tabular}

Se le preguntó al personal si un cambio en la empresa afectaría sus ingresos, las respuestas fueron: el 36\% de la población respondió casi siempre, mientras que el 27\% contestó siempre, en tanto el 23\% manifestó casi nunca y el $14 \%$ nunca. Lo cual evidencia que la mayoría de la población encuestada considera que un cambio en la empresa puede afectar sus ingresos. Esto se muestra en la Tabla 18.

Tabla 18. Un cambio en la empresa afectaría el ingreso de los Trabajadores

\begin{tabular}{|l|l|l|}
\hline Opciones & $F / A$ & $\%$ \\
\hline Siempre & 12 & 27 \\
\hline Casi siempre & 16 & 36 \\
\hline Nunca & 6 & 14 \\
\hline Casi nunca & 10 & 23 \\
\hline Total & 44 & 100 \\
\hline
\end{tabular}

Al personal se le preguntó si la empresa establecía normas muy rígidas, los resultados obtenidos fueron los siguientes: el $30 \%$ opinó nunca, mientras que el $27 \%$ contestó siempre, en tanto el $27 \%$ manifestó casi nunca y el $16 \%$ respondió casi siempre, por lo cual se concluye que la empresa no es rígida en las normas que establece. Presentado a continuación en la Tabla 19. En este sentido, Guizar, (2013), señala, que la cultura de una organización no es el resultado de generación espontánea. Una vez establecida rara vez desaparece; los elementos que refuerzan y sostienen la cultura son: las costumbres, tradiciones y forma general en que una organización cumple con su misión, lo cual se debe en gran medida a su historia y el grado de éxito conseguido, y conduce a la fuente primera de su cultura: los fundadores. Como estos son los autores de la idea original, también suelen tener prejuicios sobre la forma de alcanzar sus metas. En cuanto a la cultura fuerte, destaca que las normas y políticas son establecidas según los valores y creencias de la organización obligando a los trabajadores a compartirlas y aceptarlas, lo cual garantiza el cumplimiento de los objetivos organizacionales, pero a su vez, puede generar una actitud negativa en los trabajadores para asumir un proceso de cambio.

Tabla 19. Rigidez de la empresa en el establecimiento de sus normas

\begin{tabular}{|l|l|l|}
\hline Opciones & F/A & $\%$ \\
\hline Siempre & 12 & 27 \\
\hline Casi siempre & 7 & 16 \\
\hline Nunca & 13 & 30 \\
\hline Casi nunca & 12 & 27 \\
\hline Total & 44 & 100 \\
\hline
\end{tabular}

Sobre el asunto, Robbins y Judge (2017), exponen que mientras más fuerte sea la cultura en una organización más compromiso adquirirá el individuo y proporcionará mayor efectividad en sus labores, ya que éste se sentirá aún más comprometido con su empresa y la toma de decisiones que ejecute afectará, no sólo a la organización sino también a éste, ya que él forma parte de ella. Parra et al, sostienen que las fuerzas externas para el cambio son las que tienen su origen fuera de la organización. Dado que estas fuerzas tienen efectos globales, pueden hacer que una organización se pregunte sobre la naturaleza del negocio al que se dedica y sobre la producción de bienes y servicios; en cuanto a las fuerzas internas para el cambio tienen su origen en el seno de la organización. A su vez Simancas et al (2018), enuncian que estas fuerzas pueden ser tan sutiles como un bajo nivel de moral, o pueden manifestarse en hechos tan evidentes como la baja productividad y los conflictos internos. Las fuerzas internas pueden derivarse tanto de problemas relacionados con los recursos humanos como de las conductas y decisiones de los directivos.

\section{DISCUSIÓN FINAL}

En función de los resultados obtenidos en la presente investigación dirigida al análisis del Desarrollo integral de procesos de adaptación al cambio en pymes de servicios tecnológico, se enuncian las siguientes conclusiones: se pudo conocer que la organización actúa basada en los patrones de conducta, valores, 
normas, creencias para alcanzar sus objetivos, de igual manera los trabajadores comparten y aceptan estos elementos, lo que conduce una interacción entre ambos lo cual permite alcanzar las metas propuestas.

Se determinó que la cultura predominante es fuerte, porque, aunque se les permite a los trabajadores realizar sugerencias para tomar en cuenta las opiniones de estos, se sostienen con intensidad, se comparten los valores centrales de la organización. En este sentido la cultura organizacional de la empresa obliga a los trabajadores a adaptarse a ella, así como lo establecen Parra (2019), y de esta manera adquirir un compromiso que le proporcione una mayor efectividad en las labores, lo que constituye una ventaja, pero a su vez genera como desventaja la eliminación de las fortalezas que traen a la organización las personas con diferentes contextos organizacionales, generando así una cultura propia que se cierra ante la incorporación de nuevos elementos dentro de esta, provenientes de empleados más noveles dentro de la organización, por otro lado, los patrones de conducta se resisten a las fuerzas del cambio al correr del tiempo.

Se determinaron las fuentes de resistencia al cambio, donde las costumbres actúan como fuente principal para la resistencia al cambio, no se le ha permitido al trabajador capacitarse en cuanto a las nuevas técnicas de trabajo, así como también existe el temor de una reducción de personal o tal, vez se sientan afectados sus ingresos, en relación con estas implicaciones se concluye que la empresa no ha generado una actitud positiva en los trabajadores con respecto al proceso de cambio. En cuanto al tipo de cambio a implantar, así como lo exponen Valenzuela et al., (2018) en la región Caribe se vincula la necesidad de romper con las viejas costumbres para dar paso a las nuevas tecnologías, de igual modo la empresa cuando quiere implementar una nueva metodología de trabajo alienta al personal para que asuman el cambio en forma positiva, pero no se lleva a cabo el proceso de aprendizaje como tal que permita romper con los antiguos paradigmas dando paso al cambio y recongelamiento para cumplir con los objetivos propuestos y lograr el éxito de la organización.

\section{CONCLUSIONES}

De acuerdo al trabajo presentado y a los resultados obtenidos, se pueden plantear las siguientes conclusiones principales:

1.- Las empresas objeto de estudio deben tomar en cuenta los lineamientos referidos a la cultura organizacional, flexibilizar los procesos y acciones, en virtud de poner generar en los empleados la adaptación al cambio. Dado que en la actualidad se está utilizando la tecnología cada vez con mayor intensidad como medio para mejorar la productividad y la competitividad en los mercados.

2.- Se pudo constatar que existen fuerzas endógenas que crean la necesidad del cambio estructural y del comportamiento, que generan tensión organizacional, en las empresas del sector tecnológico, donde se resaltan tensión de las actividades, interacciones, sentimientos o resultados del desempeño en el trabajo.

3. La cultura existente en la empresa está enmarcada con paradigmas arraigados a las actitudes, valores y prioridades en la empresa, esto afecta el desarrollo de la empresa, por ende, el del empleado, la cultura existente establece ciertas limitaciones, en cuanto al plan de carrera o adiestramiento del personal, no se están aplicando nuevas técnicas gerenciales, de trabajo en equipo, por otra parte, las fuerzas externas a la empresa no permiten cubrir las necesidades del cliente.

\section{REFERENCIAS}

Bravo, S., Donado, A., y otros 3 autores, MiPyMEs asociadas y no asociadas: una aproximación a su gestión en innovación y desarrollo tecnológico, ISSN: 0798-1015, Revista Espacios, 38(58), 17-37 (2017)

Chiavenato, I., Human resources: the human capital of organizations: how to attract, apply, maintain, develop and monitor this valuable organizational treasure (2015)

Cradazco, W., Niebles, W., y otros 3 autores, Strategic Management for SMEs: For the Projection to Global Markets, doi: doi.org/10.5539/mas.v13n1p99, Modern Applied Science, 13(1), 99-105 (2019)

De la Hoz, J., Hernández, H. y otros 6 autores, Management model for the logistics and competitiveness of SMEs in the city of Barranquilla. In: Rocha, Á., Reis, J., Peter, M., Bogdanović Z., (eds) Marketing and smart technologies, doi: doi.org/10.1007/978-981-15-1564-4_37, Smart Innovation, Systems and Technologies, vol 167. Springer, Singapore (2020)

French, W., Bell, C. y Zawacki, R., Organization development and transformation: Managing effective change. Macmillan/McGraw-Hill School (2005)

García J., Durán, S., Parra, M. y Martínez, H., Inserción, integración y equidad en el ámbito laboral: Escenario empresarial post conflicto en Colombia. doi: dx.doi.org/10.31876/rcs.v24i3, Revista de Ciencias Sociales 24(3), 36-49, (2018) 
Hernández, R., Fernández, C. y Baptista, P., Metodología de la Investigación. Editorial McGraw Hill, México DF, México (2017)

Hernández, H., Cardona, D. y Pineda, M., Proyección estratégica de la calidad como facilitadora para la innovación en el sector salud de la ciudad de Región Caribe, doi: doi.org/10.22507/rli.v14n1a15, Revista Lasallista de Investigación, 14(1), 170-178 (2017)

Hernández, I., Parra, M. y otros 4 autores, Comportamiento Organizacional Ciudadano (COC) como ejemplo de participación generador de un diálogo de saberes, ISSN: 0798-1015, Revista Espacios, 39(7), 22-36 (2018)

Jiménez, J., Mojica, J., Hernández, H. y Cardona, D., Diagnóstico de la innovación y desarrollo tecnológico en el sector hotelero de la región caribe colombiana, doi: dx.doi.org/10.4067/S0718-07642018000500157, Información Tecnológica, 29(5), 157-164 (2018)

Kinicki, A. y Kreitner, R., Organizational behavior: Key concepts, skills \& best practices. Columbus, OH: McGraw-Hill/Irwin (2006)

Marulanda, C., López, L. y Cruz, G., La cultura organizacional, factor clave para la transferencia de conocimiento en los centros de investigación del triángulo del café de Colombia, doi: dx.doi.org/10.4067/S0718-07642018000600245, Información Tecnológica, 29(6), 245-252 (2018)

Mondy, R. y Martocchio, J., Human resource management. 14th Edition, Pearson (2016)

Moreno, Z., Parra, M., y otras 3 autoras., Importancia del pensamiento estratégico y acciones estratégicas para impulsar el emprendimiento social en las universidades venezolanas, ISSN: 0798-1015, Revista Espacios, 38(1) (2017)

Osorio, V., y Rodríguez, M., Modelo para la evaluación de programas de ética organizacional en universidades, doi: dx.doi.org/10.4067/S0718-07642018000100059, Información Tecnológica, 29(1), 59-70 (2018)

Parra, M., Visbal, O., Durán, S., y Badde, G., Calidad de la comunicación y actitud de los empleados ante procesos de cambio organizacional Interdisciplinaria, doi: doi.org/10.16888/interd.2019.36.1.11, Centro Interamericano de Investigaciones Psicológicas y Ciencias Afines, 36 (1), 155-170 (2019)

Pierce, J. y Newstrom, J., The manager's bookshelf: pearson new international edition. Pearson Higher Ed, (2013)

Robbins, S., y Judge T., Organizational behavior, 17th Edition, Pearson Editorial Pearson (2017)

Simancas, R., Silvera, A., Garcés, L. y Hernández, H., Administración de recursos humanos: factor estratégico de productividad empresarial en pymes de Barranquilla, doi: dx.doi.org/10.31876/revista.v23i82, Revista Venezolana de Gerencia, 23(82), 377-391 (2018)

Tarhini, A., Ammar, H., Tarhini, T. y Masa'deh, R., Analysis of the critical success factors for enterprise resource planning implementation from stakeholders' perspective: A systematic review. International Business Research, 8(4), 25-40 (2015)

Valenzuela, I., Parra M. y otros 8 autores, Factores del clima organizacional existentes en el área administrativa en empresas de región caribe, ISSN: 0798-1015, Revista Espacios, 39(50), 21-35 (2018)

Viloria, A., Martínez, D. y otros 6 autores, Optimization of flow shop scheduling through a hybrid genetic algorithm for manufacturing companies. In: Pandian A., Ntalianis K., Palanisamy R. (eds) Intelligent Computing, Information and Control Systems. ICICCS 2019. Advances in Intelligent Systems and Computing, doi: doi.org/10.1007/978-3-030-30465-2_3, Springer, Cham, Vol 1039 (2020) 
